\title{
A novel Terahertz Integrated Horn Antenna on Silicon Substrate
}

\author{
Lin $\mathrm{Guo}^{1, \mathrm{a}}$, Haixia $\mathrm{Tang}^{1}$, Lian $\mathrm{Ji}^{2}$, Yongpeng $\mathrm{Wu}^{3}$ \\ ${ }^{1}$ Key Laboratory of Broadband Wireless Communication and Sensor Network Technology, Ministry \\ of Education, College of Internet of Things, Nanjing University of Posts and Telecommunications, \\ Nanjing 210003, China; \\ ${ }^{2}$ College of Energy, Nanjing University of Technology, Nanjing 210009, China; \\ ${ }^{3}$ College of Communication and Information Engineering, Institute for Digital Communications, \\ Universitüt Erlangen-Nürnberg, Cauerstrasse 7, D-91058 Erlangen, Germany; \\ agglin5047259@163.com
}

Keywords: Terahertz (THz) wave, micro-electromechanical Systems (MEMS), silicon substrate, On-chip antenna, horn antenna

Abstract. This paper reports the design and analysis of a novel pyramidal horn antenna based on Micro Electro Mechanical Systems (MEMS) technology, which is used in terahertz (THz) applications. The structure of the antenna includes two parts, pyramidal horn structure and rectangle waveguide for feed, the fabrication of the structure needs only one wafer, and the antenna can be easily integrated with other parts of on-chip circuits. There are many tunable parameters of the horn, so that high performance $\mathrm{THz}$ antenna can be achieved by optimizing these parameters. The proposed antenna can be manufactured by MEMS processing with low fabrication cost, suitable for many terahertz systems.

\section{Introduction}

Terahertz has drawn people's attention nowadays for its' imaging systems can provide images with higher-resolution compare to microwave imaging systems, what's more, it' sable to bring less harm to human body than X-ray imaging systems. And it has been taken into many applications, for example, nondestructive evaluation and medical use [1]. Terahertz fields refer to the waves with a spectrum between 0.1 and $10 \mathrm{THz}$, which is between the microwave and the infrared regions. The domain of $\mathrm{THz}$ science locates in the transition area from macrophysics to microcosmic quantum theory [2]. For these and future applications in THz communication and surveillance, the efficient transmission (reception) of $\mathrm{THz}$ signal in (from) the free space is fundamental and suitable antenna designs are required [3-5].

Being the most important and irreplaceable components in a $\mathrm{THz}$ system, antenna is playing an important role in not only impedance match but also power radiation. The THz applications rely on the antenna elements with compact size, wide bandwidth, and high directional gain [5]. Planar antennas are used extensively in THz applications, because they can be integrated with other devices easily, such as diodes. Many THz antennas provided with varied structures have been sprung up, for example butterfly-shaped antenna, the $\mathrm{THz}$ dipole antenna [2], Micro-Lens antenna [5], bow-tie antennas [6], and skirt Antenna [6]. However, the impedance bandwidths of the dipoles are inherently relative narrow and the gains of these broadband antennas are relatively low and their geometries results in a difficulty to make them as a planar array [1-7].

Integrated antennas are very compact and suitable for low cost integration. Among the different integrated antenna designs proposed, the integrated horn antenna has some desirable characteristics for terahertz pulses radiation: very broadband, non-dispersive and relatively simple to fabricate [7-9]. One kind of integrated horn antenna designed by G. M. Rebeiz utilized a silicon pyramid cavity, which is fabricated by MEMS wet etching technique. This method is a very good solution to enhance the antenna radiation, but the design requires several wafers thereby increasing the complexity of processing and integration [9-11]. 
In this paper, we report a novel integrated MEMS pyramidal horn antenna structure, which could overcome these drawbacks. This method employs both the $\mathrm{KOH}$ etching to fabricate antenna structures and the RIE etching to fabricate rectangle waveguide structures on only one wafer, and the dimensional tolerance of this technique is determined by the lithography process, which could be controlled down to tens nano-meters. The antenna has interesting characteristics such as large bandwidth, low reflection coefficient and good directivity. Details of the antenna design are presented, and the structure is simulated by Ansoft High Frequency Structure Simulator (HFSS) 13 to verify the performance of the proposed antenna.

\section{Antenna Design and Simulation}

The size of $\mathrm{THz}$ antenna is closely related to the wavelength, which is about $30 \mu \mathrm{m}(10 \mathrm{THz})$ to $3 \mathrm{~mm}(0.1 \mathrm{THz})$, thus MEMS technology is highly suitable for manufacturing $\mathrm{THz}$ devices since nm-level precision can be achieved using this technology. The proposed antennas can be fabricated in single-crystal silicon substrates using conventional bulk micromachining.

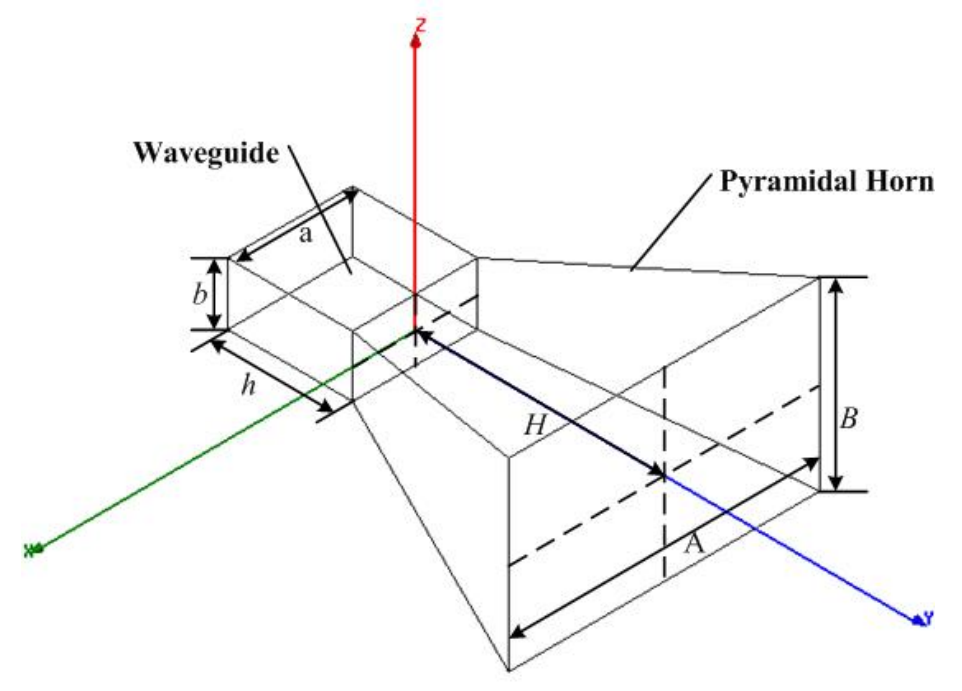

Fig. 1 Geometry of the proposed design

The process involves a sequence of wet etching and dry etching, dielectric layer deposition, and cooper plating. Fig. 1 shows geometrical configuration of the proposed antenna, which can be determined by the configuration of etched cavity. The geometrical parameters can be determined by following equation:

$$
H=\frac{A-a}{2} \times \tan \alpha=\frac{B-b}{2} \times \tan \alpha
$$

The geometrical parameters along $\mathrm{x}$-axis and $\mathrm{y}$-axis can be determined by Eq.1 independently.

\section{Results and Discussion}

Table 1 Dimensions of the Proposed Antenna

\begin{tabular}{cccc}
\hline Parameters & Values $(\mu \mathrm{m})$ & Parameters & Values $(\mu \mathrm{m})$ \\
\hline A & 890 & $\mathrm{H}$ & 450 \\
$\mathrm{a}$ & 254 & $\mathrm{~h}$ & 100 \\
$\mathrm{~B}$ & 763 & Parameters & Values $\left(^{\circ}\right)$ \\
$\mathrm{b}$ & 127 & $\alpha$ & 54.7 \\
\hline
\end{tabular}

We use Ansoft High Frequency Structure Simulator (HFSS) 13 to analyze the performance of the proposed structure. A set of optimized antenna parameters are shown in Table 1. The simulation results were shown in Fig. 2-4. 


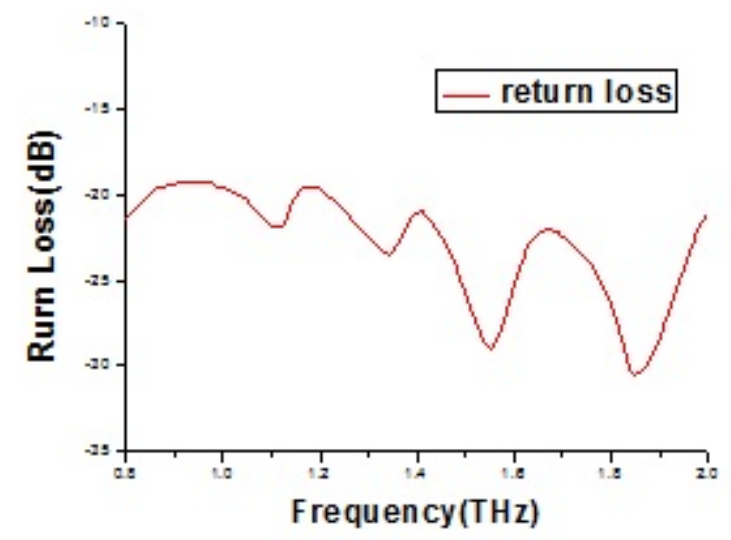

Fig.2 Simulated return loss of the antenna.

Fig. 2 shows the simulated return loss for the antenna. It is clear that the return loss of the pyramidal horn antenna stays below $-20 \mathrm{~dB}$ across the interest frequency band of $0.8-2 \mathrm{THz}$.
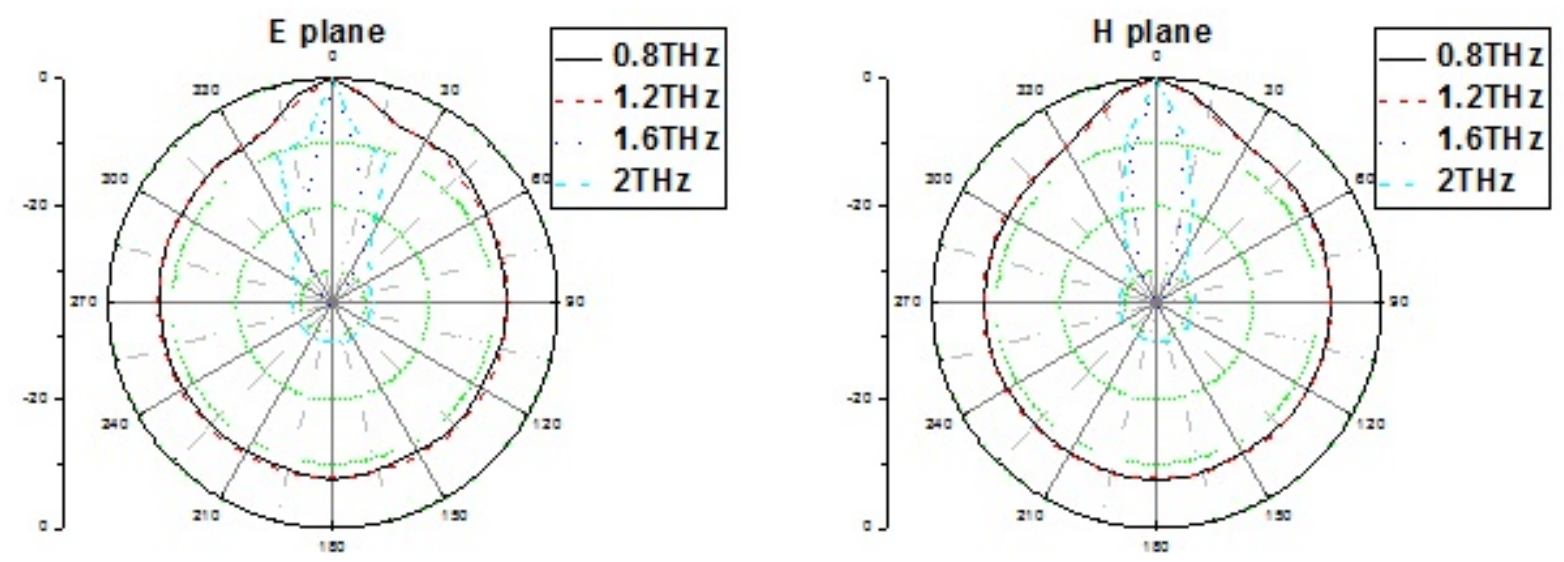

Fig.3 Simulated radiation patterns of the pyramidal horn antenna at different pass band frequencies.

The radiation patterns at xoz-plane (E-plane) and yoz-plane (H-plane) of the pyramidal horn antenna simulated at some in-band frequencies are plotted in Fig.3. These frequencies chosen are located at $0.8 \mathrm{THz}, 1.2 \mathrm{THz}, 1.6 \mathrm{GHz}, 2 \mathrm{THz}$, in the pass band of this antenna. As this figure indicates, both in E-plane and H-plane, the radiation patterns keep good directivity, and as the frequency increases, the orientation is improved.

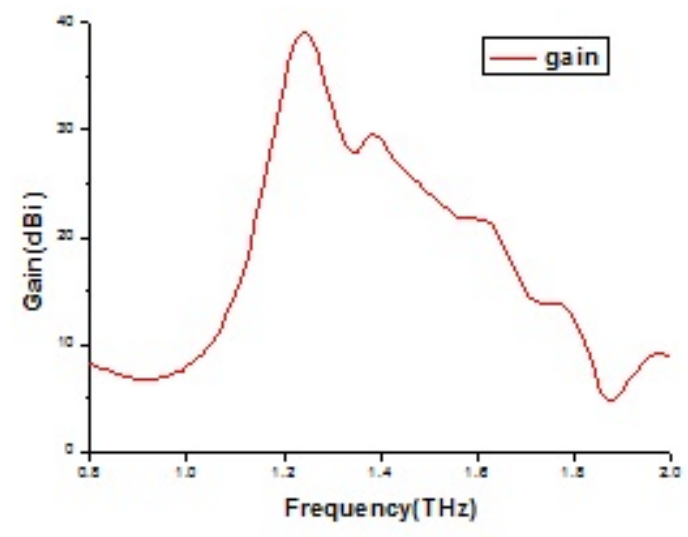

Fig.4 Simulated gain of the antenna.

Fig.4 shows the simulated gain of the pyramidal horn antenna. The average simulated gain of the antenna is about $18 \mathrm{~dB}$. 


\section{Summary}

As new applications for terahertz instruments begin to emerge, a new generation of high efficiency antennas and antenna systems must be developed to meet the specific requirements of these high frequency systems. In this paper, the integrated MEMS pyramidal horn antenna has been proposed and investigated. This design shows a wideband input match, stable radiation patterns, and smooth gain responses. These characteristics and the simple, compact, low cost, and easy integration make the proposed pyramidal horn antenna highly suitable for the $\mathrm{THz}$ application systems.

\section{Acknowledgments}

This work is sponsored by National Natural Science Foundation of China (Grant No. 61404157), the Research Fund of ZTE Corporation and the NUPTSF (Grant No. NY214189 and No. NY215146 ). The corresponding author is Lin Guo, gglin5047259@163.com.

\section{References}

[1] N. Zhu, R. W. Ziolkowski, "Photoconductive THz Antenna Designs With High Radiation Efficiency High Directivity and High Aperture Efficiency”, IEEE Trans. Terahertz Sci. Technol., vol.3, no.6, pp.721-730, Novermber 2013.

[2] I.C.Mayorga,A.Schmitz,T.Klein,C.Leinz,andR.Gusten,"First in-field application of a full photonic local oscillator to terahertz astronomy,” IEEE Trans. Terahertz Sci. Technol., vol. 2, no. 4, pp.393-399,Jul.2012.

[3] L. Prissette, G. Ducournau, T. Akalin, E. Peytavit, A. Beck, M. Zaknoune, D. Ducatteau, and J.F Lampin, in: IEEE Microwave and Wireless Components Letters, vol. 21, no. 1, (2011)pp. 49 51.

[4] Y. Wu, C. Xiao, Z. Ding, X. Gao, and S. Jin, "Linear precoding for finite alphabet signaling over MIMOME wiretap channels," IEEE Trans. Veh. Technol., vol. 61, no. 6, pp. 2599-2612, July 2012.

[5] M. Alonso-DelPino, N. Llombart, G. Chattopadhyay, C. Lee, C. Jung-Kubiak, L. Jofre, and I. Mehdi “Design Guidelines for a Terahertz Silicon Micro-Lens Antenna,” IEEE Antennas and Wireless Propagation Letters, vol.12, pp84-87, 2013.

[6] H. Liu, J. Yu, P. Huggard, et al. A multichannel THz detector using integrated Bow-Tie antennas[J]. International Journal of Antennas and Propagation, 2013

[7] N. Kaminski, D. Corcos, E. Shumaker, et al. Wideband planar skirt antenna and its application for the detection of terahertz radiation[C]//Microwaves, Communications, Antennas and Electronics Systems (COMCAS), 2013 IEEE International Conference on. IEEE, 2013: 1-4.

[8] L. Marnat, A. Arevalo Carreno, D. Conchouso, et al. New movable plate for efficient millimeter wave vertical on-chip antenna[J]. 2013.

[9] B. Chen, V. N. Sekhar, C. Jin, et al. Low-Loss Broadband Package Platform With Surface Passivation and TSV for Wafer-Level Packaging of RF-MEMS Devices[J]. 2013.

[10]G.V. Eleftheriades, W.Y. Ali-Ahmad, and G.M. Rebeiz, in: IEEE Microwave and Wireless Components Letters, vol. 2, no. 2, (1992), pp. 49 - 51.

[11]G. M. Rebeiz, L. P. Katehi, W. Y. Ali-Ahmad, G. V. Elefheriades, and C. C. Ling, in: IEEE Antennas and Propagation Magazine, vol. 34, no. 1, (1992), pp. 7-16. 